\title{
Analytic structure of a family of hyperboloidal beams of potential interest for advanced LIGO
}

\author{
Vincenzo Galdi, ${ }^{*}$ Giuseppe Castaldi, Vincenzo Pierro, and Innocenzo M. Pinto \\ Waves Group, Department of Engineering, University of Sannio, I-82100 Benevento, Italy \\ Juri Agresti, Erika D’Ambrosio, and Riccardo DeSalvo \\ LIGO Laboratory, California Institute of Technology, Pasadena, California 91125, USA
}

(Received 20 February 2006; published 22 June 2006)

\begin{abstract}
This paper is concerned with a study of the analytic structure of a family of hyperboloidal beams introduced by Bondarescu and Thorne which generalizes the nearly-flat and nearly-concentric mesa beam configurations of interest for advanced LIGO. Capitalizing on certain results from the applied optics literature on flat-top beams, a physically-insightful and computationally-effective representation is derived in terms of rapidly-converging Gauss-Laguerre expansions. A generalization (involving fractional Fourier transform operators of complex order) of some recently discovered duality relations between the nearly-flat and nearly-concentric mesa configurations is obtained. Possible implications for the advancedLIGO optical cavity design are discussed.
\end{abstract}

DOI: 10.1103/PhysRevD.73.127101

PACS numbers: 07.60.Ly, 04.80.Cc, 41.85.Ew, 42.55.-f

\section{INTRODUCTION}

Fabry-Perot optical cavities with nonspherical mirrors capable of supporting flat-top ("mesa") beams are being actively investigated [1] to be used in the baseline design of the advanced Laser Interferometer Gravitational-wave Observatory (LIGO) [2]. These configurations may reduce the thermal noise of the mirrors through better averaging over the beam profile of the thermally-induced surface fluctuations $[3,4]$. In this framework, nearly-flat, "Mexican-hat-shaped" mirror configurations were found capable of providing a reduction by a factor three in the thermoelastic noise power and a factor two in the coating Brownian thermal-noise power [5], without substantial fabrication impediments. To reduce the severe tiltinstability problems [6] affecting this originally conceived configuration [7], K.S. Thorne proposed a nearlyconcentric mirror configuration capable of producing the same mesa beam profile on the mirror surfaces, but featuring a much weaker tilt-instability. Remarkably, the nearlyflat (FM) and nearly-concentric mesa (CM) configurations were found to be connected through a duality relation [7,8], which allows a one-to-one mapping between all the corresponding eigenmodes. The geometrical construction underlying FM and CM beams [3], based on coherent superposition of minimum-spreading Gaussian beams (GBs), was further generalized by Bondarescu and Thorne [9], in terms of a one-parameter family of "hyperboloidal" beams which allows continuous spanning from the FM to the CM configurations, and including the standard GB case.

This paper elaborates on the analytic structure of the Bondarescu-Thorne (BT) hyperboloidal beams, building on a number of results from the applied optics literature

\footnotetext{
*Electronic address: vgaldi@unisannio.it; http://www.ing.unisannio.it/vgaldi
}

concerning flat-top beams, which have most likely not come to the attention of the gravitational-wave community. Specifically, the FM and CM beams belong to the class of flattened beams introduced in [10], and can therefore be represented in terms of the rapidly-converging GaussLaguerre (GL) beam expansions derived therein. Based on this observation, we extend the approach in [10] to accommodate the more general family of BT hyperboloidal beams [9]. This leads to a generalization (we limit the analysis here to the dominant eigenmode) of the FM-CM duality relations in $[7,8]$, which involves fractional Fourier transforms of complex order.

\section{BT HYPERBOLOIDAL BEAMS}

Referring to the problem geometry illustrated in Fig. 1, we consider a perfectly symmetric Fabry-Perot optical cavity composed of two nearly-spheroidal mirrors separated by a distance $L$ along the $z$-axis of a Cartesian $(x, y, z)$ (and associated cylindrical $(r, \theta, z)$ ) coordinate system. The transverse coordinates at the waist $(z=0)$ and mirror $(z=L / 2)$ planes are denoted by $\mathbf{r}_{0} \equiv x_{0} \hat{\mathbf{x}}+y_{0} \hat{\mathbf{y}}=$ $r_{0} \cos \theta_{0} \hat{\mathbf{x}}+r_{0} \sin \theta_{0} \hat{\mathbf{y}} \quad$ and $\quad \mathbf{r} \equiv x \hat{\mathbf{x}}+y \hat{\mathbf{y}}=r \cos \theta \hat{\mathbf{x}}+$ $r \sin \theta \hat{\mathbf{y}}$, respectively. Here and henceforth, $\hat{\mathbf{x}}, \hat{\mathbf{y}}$ and $\hat{\mathbf{z}}$ denote the standard Cartesian unit vectors. Throughout the paper, an implicit time-harmonic $\exp (-i \omega t)$ dependence is assumed for all field quantities.

The BT construction [9], which generalizes the original idea in [3], is based on the superposition of minimumspreading GBs launched from a circular equivalent aperture of radius $R_{0}$ at the waist plane $(z=0)$, with optical axes pointing along the unit vector $\mathbf{u}_{\alpha}=\left(r_{0} / L\right)\left[\cos \theta_{0}-\right.$ $\left.\cos \left(\theta_{0}-\alpha\right)\right] \hat{\mathbf{x}}+\left(r_{0} / L\right)\left[\sin \theta_{0}-\sin \left(\theta_{0}-\alpha\right)\right] \hat{\mathbf{y}}+\hat{\mathbf{z}}$. These optical axes are readily recognized to be the generators of hyperboloids (see, e.g, [9], Fig. 1(b)). The "twist-angle" $\alpha$ parameterizes this family of hyperboloidal beams, allowing continuous spanning from the FM configuration 


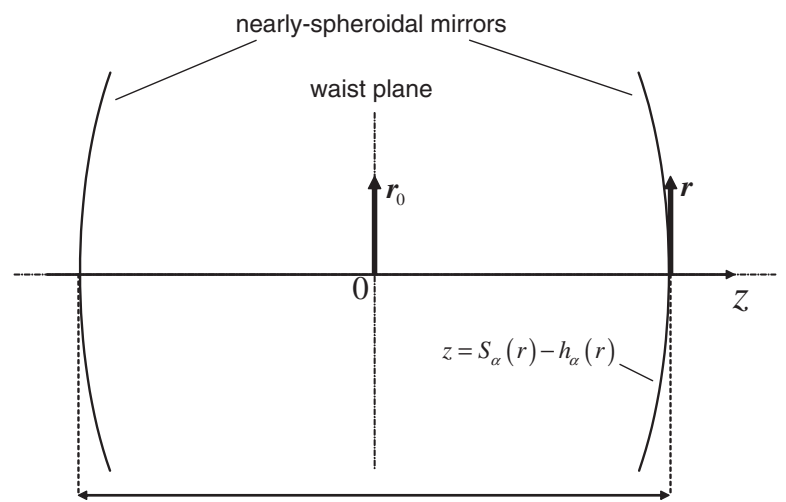

$L$

FIG. 1. Sketch of the problem geometry (see text).

( $\alpha=0$, cylindrical degenerate, cf. [9], Fig. 1(a)) to the CM configuration ( $\alpha=\pi$, conical degenerate, cf. [9], Fig. 1(c)). For this family of beams, the wavefronts at the mirror location are roughly approximated by the "fiducial" spheroids [9]

$$
z=S_{\alpha}(r) \equiv \sqrt{\left(\frac{L}{2}\right)^{2}-r^{2} \sin ^{2}(\alpha / 2)},
$$

which degenerate into planar and spherical surfaces in the FM $(\alpha=0)$ and CM $(\alpha=\pi)$ case, respectively. Following [9], an integral expression (valid under the paraxial approximation) for the (unnormalized) beam field distribution on these fiducial surfaces can be written as

$$
\begin{aligned}
U_{\alpha}\left(r, S_{\alpha}\right)= & \Lambda \int_{0}^{R_{0}} d r_{0} \int_{0}^{2 \pi} d \theta_{0} r_{0} \exp \left[i \frac{r r_{0}}{w_{0}^{2}} \sin \theta_{0} \sin \alpha\right. \\
& \left.-\frac{\left(r^{2}+r_{0}^{2}-2 r r_{0} \cos \theta_{0}\right)}{2 w_{0}^{2}}(1-i \cos \alpha)\right] .
\end{aligned}
$$

In (2), $\Lambda$ is an $\alpha$-independent complex constant, and the GB spot size at the waist is chosen as $w_{0}=\sqrt{L / k_{0}}\left(k_{0}=\right.$ $2 \pi / \lambda_{0}$ denoting the free-space wavenumber, and $\lambda_{0}$ the free-space wavelength), so that the mirror plane is located exactly at the Rayleigh distance [11], $z_{R} \equiv k_{0} w_{0}^{2} / 2=$ $L / 2$. For $\alpha=\pi / 2$, the double integral in (2) can be computed in closed form, yielding a simple Gaussian [9]. For other values of $\alpha$, the radial integral in (2) can still be computed analytically, while the angular integral has to be evaluated numerically.

In order for the optical cavity to support a stable beam with a given profile as the fundamental eigenmode, its mirror profile has to match the beam wavefront; this can be achieved by applying a correction [12]

$$
h_{\alpha}(r)=\frac{\arg \left[U_{\alpha}\left(r, S_{\alpha}\right)\right]-\arg \left[U_{\alpha}\left(0, S_{\alpha}\right)\right]}{k_{0}}
$$

to the fiducial spheroidal shape $S_{\alpha}$ in (1), which for the FM $(\alpha=0)$ and CM $(\alpha=\pi)$ cases reduces to the Mexicanhat-shaped profile in [4] [see also Fig. 3(a) below). Moreover, from (2), the remarkable result

$$
h_{\pi-\alpha}(r)=-h_{\alpha}(r)
$$

follows, which can also be interpreted within the duality framework developed in $[7,8]$.

\section{ANALYTIC STRUCTURE OF BT BEAMS}

Our approach is aimed at finding a GL beam expansion for a generic BT hyperboloidal beam

$$
U_{\alpha}(r, z)=\sum_{m=0}^{\infty} A_{m}^{(\alpha)} \Psi_{m}(r, z)
$$

where $A_{m}^{(\alpha)}$ are $\alpha$-dependent expansion coefficients to be determined, and $\Psi_{m}(r, z)$ denote the standard GL beam propagators [11]

$$
\begin{aligned}
\Psi_{m}(r, z)= & \frac{w_{0}}{w(z)} \psi_{m}\left[\frac{\sqrt{2} r}{w(z)}\right] \exp \left[i \frac{k_{0} r^{2}}{2 R(z)}\right] \\
& \times \exp \left\{i\left[k_{0} z-(2 m+1) \Phi(z)\right]\right\} .
\end{aligned}
$$

In (6), $w(z), R(z)$ and $\Phi(z)$ denote the standard GB spot size, wavefront radius of curvature, and Gouy phase, respectively [11]

$$
\begin{gathered}
w(z)=w_{0} \sqrt{1+\left(\frac{z}{z_{R}}\right)^{2}}, \quad R(z)=z+\frac{z_{R}^{2}}{z}, \\
\Phi(z)=\arctan \left(\frac{z}{z_{R}}\right),
\end{gathered}
$$

and $\psi_{m}(\xi)$ are orthonormal GL basis functions,

$$
\psi_{m}(\xi)=\sqrt{2} \exp \left(-\frac{\xi^{2}}{2}\right) L_{m}\left(\xi^{2}\right)
$$

with $L_{n}(\zeta)$ denoting an $n$ th-order Laguerre polynomial [[13], Chap. 22]. The computation of the expansion coefficients can be addressed by recalling the field distributions at the waist plane $(z=0)$ for the CM $(\alpha=\pi)$ and FM $(\alpha=0)$ beams, which were shown [8] to be related via Fourier transform,

$$
\begin{aligned}
& U_{\pi}(r, 0)=\frac{w_{0}^{2}}{r R_{0}} J_{1}\left(\frac{2 r R_{0}}{w_{0}^{2}}\right) \exp \left(-\frac{r^{2}}{w_{0}^{2}}\right), \\
& U_{0}(r, 0)=\frac{2}{R_{0}^{2}} \int_{0}^{R_{0}} d r_{0} r_{0} I_{0}\left(\frac{2 r r_{0}}{w_{0}^{2}}\right) \exp \left[-\frac{\left(r^{2}+r_{0}^{2}\right)}{w_{0}^{2}}\right] .
\end{aligned}
$$

Here and henceforth, $J_{n}(\xi)$ and $I_{n}(\xi)$ denote $n$-order standard and modified Bessel functions of the first kind [[13], Chap. 9], respectively. The CM and FM field distributions in (9) are recognized to coincide with the so-called "flattened" and "inverse flattened" beams introduced in [10], for which a GL beam expansion was subsequently derived. Following [10], the corresponding expansion coefficients in (5) are given by

$$
\begin{aligned}
A_{m}^{(\pi)} & =\frac{\sqrt{2} w_{0}^{2}}{R_{0}^{2}} P\left(m+1, \frac{R_{0}^{2}}{2 w_{0}^{2}}\right), \\
A_{m}^{(0)} & =(-1)^{m} A_{m}^{(\pi)},
\end{aligned}
$$

where $P(n, \xi)$ denotes an incomplete Gamma function 
[[13], Eq. (6.5.13)]. The $A_{m}^{(\pi)}$ are almost constant for $m \lesssim$ $R_{0}^{2} /\left(2 w_{0}^{2}\right)$, and fall off quite rapidly for $m \geqslant R_{0}^{2} /\left(2 w_{0}^{2}\right)$ (see [10], Fig. 2). For the parametric range of interest for advanced LIGO $\left(w_{0} / R_{0}=0.25\right)$, this results in a rapidlyconverging $(m<20)$ expansion (5). A natural question then arises, as to whether the FM-CM expansion coefficient relation (10b) can be generalized to arbitrary values of the twist-angle $\alpha$, thereby allowing a GL representation for general BT hyperboloidal beams. In this connection, one notes that the mapping

$$
A_{m}^{(\alpha)}=(-\cos \alpha)^{m} A_{m}^{(\pi)}
$$

accounts correctly for the three notable cases $\alpha=0$ (FM), $\alpha=\pi / 2$ (GB) and $\alpha=\pi(\mathrm{CM})$. One is accordingly led to speculate whether it may hold for arbitrary values of the twist angle $\alpha$. This turns out to be indeed the case, as verified below by numerical comparison against the BT reference solution in (2). In all examples below, the relevant parameters (specified in the figure captions) were chosen as in [4,9]. For the truncation of the GL series involved, a simple criterion was utilized, requiring that the magnitude of the last retained $M$-th term is less than $0.1 \%$ of that of the leading term, $\left|A_{M}^{(\alpha)}\right|<10^{-3}\left|A_{0}^{(\alpha)}\right|$. For the cases $\alpha=0, \pi$ this yields $M=18$. In view of the coefficient mapping in (11), the convergence becomes faster as $\alpha$ approaches the critical value of $\pi / 2$ (pure $\mathrm{GB}$, for which one obtains only one nonzero coefficient). As a reference solution, the BT integral representation in (2) was considered, with the radial integral computed
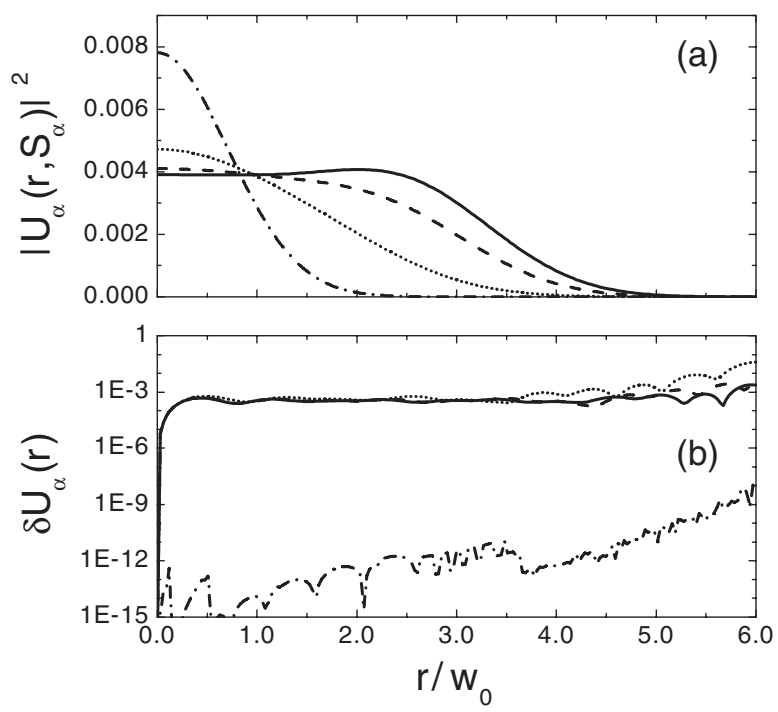

FIG. 2. BT hyperboloidal beam field distribution evaluated on the fiducial surface $z=S_{\alpha}(r)$, for different values of the twistangle parameter $\alpha$. Optical cavity parameters: $L=4 \mathrm{~km}, \lambda_{0}=$ $1064 \mathrm{~nm}, \quad w_{0}=\sqrt{L \lambda_{0} /(2 \pi)}=2.603 \mathrm{~cm}, \quad$ and $R_{0}=4 w_{0}=$ $10.4 \mathrm{~cm}$. (a) GL-computed intensity distribution. (b) Relative error $\delta U_{\alpha}$. Continuous curve: $\alpha=0, \pi(M=18)$; Dashed curve $\alpha=0.1 \pi, 0.9 \pi(M=17)$; Dotted curve: $\alpha=0.2 \pi, 0.8 \pi(M=$ 14); Dotted-dashed curve: $\alpha=0.5 \pi(M=0)$. analytically, the angular integration performed numerically, and the complex constant $\Lambda$ determined by enforcing the matching with the GL expansion at $r=0$.

Some representative results for the field distribution are shown in Fig. 2. Specifically, Fig. 2(a) shows the GLcomputed (via (5)) intensity distribution on the fiducial surface, for various values of the twist-angle $\alpha$, illustrating the gradual transition from the Gaussian $(\alpha=\pi / 2)$ to the mesa $(\alpha=0, \pi)$ profile. To quantify the agreement with the reference solution, Fig. 2(b) shows the relative error, which, consistently with the truncation criterion adopted, never exceeds $0.1 \%$ over the region of significant field intensity, and drops below numerical precision for the $\alpha=$ $\pi / 2$ (pure GB case). The results pertaining to the mirror profiles are shown Fig. 3. Specifically, Fig. 3(a) shows the GL-computed corrections $h_{\alpha}$ in (3), illustrating the gradual transition from the spherical $(\alpha=\pi / 2)$ to the Mexicanhat $(\alpha=0, \pi)$ mirror profile. Figure 3(b) shows the absolute error with respect to the reference solution, which never exceeds $10^{-4} \lambda_{0}$ over the significantly illuminated portion of the mirror. For the LIGO design $\left(\lambda_{0}=\right.$ $1064 \mathrm{~nm}$ ), this corresponds to errors $\sim 0.1 \mathrm{~nm}$, well within the typical fabrication tolerances.

The above results validate the analytic GL expansion in (5), which is obtained here for the first time, to the best of our knowledge, and sets the stage for a generalization, to arbitrary twist-angles, of the duality relations in $[7,8]$. In this framework, we consider a class of $\sigma$-parameterized generalized Hankel transform (HT) operators defined as

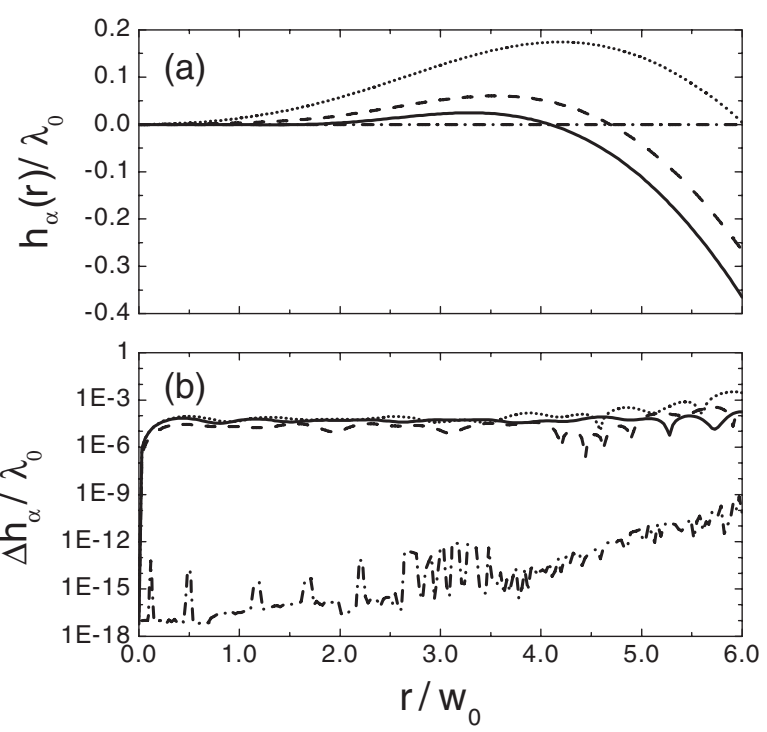

FIG. 3. Parameters as in Fig. 2. (a) Correction $h_{\alpha}$ to the fiducial spheroid $S_{\alpha}$ in (1), GL-computed via (3). (b) Absolute error $\Delta h_{\alpha}$. Continuous curve: $\alpha=\pi(M=18)$; Dashed curve: $\alpha=$ $0.9 \pi(M=17)$; Dotted curve: $\alpha=0.8 \pi(M=14)$; Dotteddashed curve: $\alpha=0.5 \pi(M=0)$. The correction profiles pertaining to $\alpha=0,0.1 \pi, 0.2 \pi$ (not shown) differ merely by sign from those pertaining to $\alpha=\pi, 0.9 \pi, 0.8 \pi$, respectively, (see (4)). 


$$
\begin{aligned}
\mathcal{H}_{w_{0}}^{(\sigma)}[F(r)] \equiv & \frac{4}{w_{0}^{2}(1+\sigma)} \int_{0}^{\infty} r_{0} d r_{0} F\left(r_{0}\right) J_{0}\left[\frac{4 r r_{0} \sqrt{\sigma}}{w_{0}^{2}(1+\sigma)}\right] \\
& \times \exp \left[-\frac{\left(r^{2}+r_{0}^{2}\right)(1-\sigma)}{w_{0}^{2}(1+\sigma)}\right], \quad \sigma \geq-1 .
\end{aligned}
$$

For $\sigma<-1$, the integral in (12a) diverges for the beams of interest here (decaying as $O\left[\exp \left(-r^{2} / w_{0}^{2}\right)\right]$ in the waist plane), and the following definition should be used:

$$
\begin{aligned}
\mathcal{H}_{w_{0}}^{(\sigma)}[F(r)] & \equiv \mathcal{H}_{w_{0}}^{(-\sigma)}\left\{\mathcal{H}_{w_{0}}^{(1)}[F(r)]\right\} \\
& =\mathcal{H}_{w_{0}}^{(1)}\left\{\mathcal{H}_{w_{0}}^{(-\sigma)}[F(r)]\right\}, \quad \sigma<-1 .
\end{aligned}
$$

The generalized HT operator in (12) can be shown (see [14], p. 43] and [15] for more details) to admit as eigenfunctions the GL basis functions in (8),

$$
\mathcal{H}_{w_{0}}^{(\sigma)}\left[\psi_{m}\left(\frac{\sqrt{2} r}{w_{0}}\right)\right]=(-\sigma)^{m} \psi_{m}\left(\frac{\sqrt{2} r}{w_{0}}\right) .
$$

Application of the generalized HT (12) to the GL expansion in (5) reveals, via (13), the functional relation between the field distributions at the waist plane pertaining to two BT hyperboloidal beams characterized by generic values, $\alpha_{1}$ and $\alpha_{2}$, of the twist-angle,

$$
U_{\alpha_{2}}(r, 0) \stackrel{\mathcal{H}_{w_{0}}^{(\sigma)}}{\longrightarrow} U_{\alpha_{1}}(r, 0), \quad \sigma=-\frac{\cos \alpha_{2}}{\cos \alpha_{1}} .
$$

The generalized HT in (14) extends, for the dominant eigenmode, the FM-CM duality relations in $[7,8]$ to the most general case, and admits a suggestive analytic inter- pretation in terms of a fractional Fourier operator of complex order [16-18] (see also [15] for more details),

$$
\gamma \equiv 1+i \frac{\log (\sigma)}{\pi}
$$

whose real part can either be 1 or 0 , whereas the imaginary part is generally nonzero (except for the cases $\alpha_{1}=\alpha_{2}$ and $\alpha_{1}=\pi-\alpha_{2}$ for which the generalized HT operator in (12) reduces to the identity and ordinary HT operator, respectively).

\section{CONCLUDING REMARKS}

In this paper, the analytic structure of a family of hyperboloidal beams of interest for advanced LIGO has been investigated, via the development of rapidly-converging GL beam expansions and a complex-Fourier-transformbased generalization of the duality relations in $[7,8]$ for the dominant eigenmode. Extension to higher-order eigenmodes, aimed at the full generalization (for arbitrary twistangles) of the duality relations in $[7,8]$ is currently under investigation. It is hoped that the above results, which provide a physically-insightful and computationallyeffective parameterization of the beam and mirror profiles, may be useful in addressing the optimization of the advanced-LIGO optical cavities in a broader perspective including a thorough parametric analysis of BT and other classes of flat-top beams (see, e.g., [19-21]), aimed at finding optimal design criteria in terms of thermal-noise and tilt-instability reduction.

\section{ACKNOWLEDGMENTS}

The work of J. A., E. D'A., and R. DS. is supported by the National Science Foundation under Grant No. PHY0107417.
[1] J. Agresti et al., LIGO Report No. LIGO-G040412-00-D, 2004 (unpublished).

[2] D. Shoemaker, LIGO Report No. LIGO-M030023-00, 2003 (unpublished).

[3] E. D'Ambrosio et al., LIGO Report No. LIGO-G00022300-D, 2000 (unpublished).

[4] E. D'Ambrosio, Phys. Rev. D 67, 102004 (2003).

[5] E. D'Ambrosio et al., gr-qc/0409075.

[6] J. A. Sidles and D. Sigg, Phys. Lett. A 354, 167 (2006).

[7] P. Savov and S. Vyatchanin, gr-qc/0409084.

[8] J. Agresti et al., gr-qc/0511062.

[9] M. Bondarescu and K.S. Thorne, gr-qc/0409083.

[10] C. J. R. Sheppard and S. Saghafi, Opt. Commun. 132, 144 (1996).

[11] A. E. Siegman, Lasers (University Science Books, Mill Valley, CA, 1986).
[12] C. Paré and P. A. Bélanger, IEEE J. Quantum Electron. 30, 1141 (1994).

[13] M. Abramowitz and I. A. Stegun, Handbook of Mathematical Functions (Dover, New York, 1964).

[14] A. Erdélyi, M. F. Oberhettinger, and F. G. Tricomi, Tables of Integral Transforms (McGraw-Hill, New York, 1954), Vol. 2.

[15] V. Galdi et al., gr-qc/0602074.

[16] L. M. Bernardo and O. D. D. Soares, Appl. Opt. 35, 3163 (1996).

[17] C. Wang and B. Lü, Optik (Jena) 113, 337 (2002).

[18] C. Wang and B. Lü, Opt. Commun. 203, 61 (2002).

[19] F. Gori, Opt. Commun. 107, 335 (1994).

[20] A. A. Tovar, J. Opt. Soc. Am. A 18, 1897 (2001).

[21] Y. Li, Opt. Lett. 27, 1007 (2002). 